\title{
SOME EXAMPLES OF GENERALIZED ZETA REGULARIZED PRODUCTS
}

\author{
Kazufumi Kimoto, Nobushige Kurokawa*, Chie Sonoki and \\ MASATO WAKAYAMA**
}

\section{Introduction and preliminaries} by

For a given sequence $\boldsymbol{a}=\left\{a_{n}\right\}_{n \in I}$, the zeta regularized product of $\boldsymbol{a}$ is defined

$$
\prod_{n \in I} a_{n}:=\exp \left(-\zeta_{\boldsymbol{a}}^{\prime}(0)\right),
$$

when the zeta function $\zeta_{\boldsymbol{a}}(s):=\sum_{n \in I} a_{n}^{-s}$ attached to the sequence $\boldsymbol{a}$ is analytically continued to some region containing the origin $s=0$ and holomorphic at the origin (see e.g., [18], [2]). This notion allows us to carry out various renormalization calculations of "divergent products". For example, we have

$$
\prod_{n=1}^{\infty} n=“ 1 \times 2 \times 3 \times 4 \times \cdots "=\exp \left(-\zeta^{\prime}(0)\right)=\sqrt{2 \pi}
$$

from Riemann's calculation $\zeta^{\prime}(0)=-(1 / 2) \log (2 \pi)$, where $\zeta(s)=\sum_{n=1}^{\infty} n^{-s}$ denotes the Riemann zeta function. The zeta regularized products are very important for the study of the various zeta functions, for instance, the determinant expressions of the Selberg zeta functions via the hyperbolic Laplacian (see for e.g., the references in [18]), and the cohomological study of the motivic $L$ functions [2].

We first remark that the following elementary operations of regularized products follow easily from the definition:

$$
\begin{gathered}
\prod_{n \in I \amalg J} a_{n}=\prod_{n \in I} a_{n} \prod_{n \in J} a_{n}, \\
\prod_{n \in I} a_{n}^{k}=\left(\prod_{n \in I} a_{n}\right)^{k},
\end{gathered}
$$

* Partially supported by Grant-in-Aid for Exploratory Research No. 15654003.

** Partially supported by Grant-in-Aid for Scientific Research (B) No. 11440010, and by Grantin-Aid for Exploratory Research No. 13874004.

Received February 24, 2004. 


$$
\prod_{n \in I} \tau a_{n}=\tau^{\zeta a}(0) \prod_{n \in I} a_{n}
$$

There are many reasons why we are interested in the zeta regularization. Among them, we now focus our attention on the particular feature that the expression of a function by the zeta regularized product respects a structural aspect of the function. For example, Lerch's formula [3]

$$
\frac{1}{\Gamma(x)}=\frac{1}{\sqrt{2 \pi}} \prod_{n=0}^{\infty}(n+x)
$$

which is the generalization of Riemann's result (1.2), gives a "factorization" of the gamma function $\Gamma(x)$, and hence it exhibits the information of the location and the multiplicity of zeros in an apparent manner. This is comparable with a role of the Weierstrass canonical form of the entire function $1 / \Gamma(x)$ :

$$
\frac{1}{\Gamma(x)}=e^{\gamma x} x \prod_{n=1}^{\infty}\left(1+\frac{x}{n}\right) e^{-x / n} .
$$

Actually, in [18], a relation between the regularized product and the canonical form is clarified in general. Further, the property (1.3) yields the basic functional equation $\Gamma(x+1)=x \Gamma(x)$. Beside these facts, it is remarkable also that the duplication formula

$$
\Gamma(2 x)=\frac{2^{2 x}}{2 \sqrt{\pi}} \Gamma(x) \Gamma\left(x+\frac{1}{2}\right)
$$

can be immediately derived from Lerch's formula by using (1.3) and (1.5) (see Example 3.9).

Another typical example is seen in the ring sine functions introduced in [9]. Let $\tau$ be an imaginary quadratic integer. In [9], the ring sine function of $\boldsymbol{Z}[\tau]$ is realized by the zeta regularized product, and calculated as follows:

$$
\begin{aligned}
S(x ; \tau) & :=\prod_{m, n \in \boldsymbol{Z}}(m+n \tau-x) \\
& =\left(1-e^{2 \pi i x}\right) \prod_{n=1}^{\infty}\left(1-e^{2 \pi i(n \tau+x)}\right)\left(1-e^{2 \pi i(n \tau-x)}\right) \quad(0<\operatorname{Im}(x)<\operatorname{Im}(\tau)) .
\end{aligned}
$$

This product gives essentially the elliptic theta function $\vartheta_{1}(x ; \tau)$. The translation invariance $S(x+1 ; \tau)=S(x ; \tau)$ is obvious in this expression. Moreover, if we denote by $\varphi(s)=\varphi(s ; \tau, x)$ the attached zeta function for $S(x ; \tau)$, we see from (1.5) that

$$
S(x ; \tau)=\tau^{\varphi(0)} \prod_{m, n \in \boldsymbol{Z}}\left(\frac{m}{\tau}+n-\frac{x}{\tau}\right)=\tau^{\varphi(0)} S(x / \tau ; 1 / \tau),
$$

which asserts substantially the transformation formula for $\vartheta_{1}(x ; \tau)$. Note that we have $\varphi(0)=-(\tau-1)^{2} / \tau$ from the calculation in [9]. Thus a zeta regularized 
product expression is expected to indicate various invariance and/or periodicity of a function in a considerably obvious way.

In contrast to the advanced feature as we explained above, the definition of a zeta regularized product does not work even in a simple case, for instance, the case $\boldsymbol{a}=\left\{q^{n}\right\}_{n \geq 0}(q>1)$, because the attached zeta function $\zeta_{\boldsymbol{a}}(s)=\sum_{n=0}^{\infty} q^{-n s}=$ $\left(1-q^{-s}\right)^{-1}$ has a simple pole at $s=0$. In order to deal with such cases, we have extended the notion of the regularized product in [13] as follows: If $\zeta_{\boldsymbol{a}}(s)$ is analytically continued to some region containing the origin $s=0$ and has a pole at the origin, then we say $\boldsymbol{a}$ is regularizable, and the (generalized) zeta reguralized product of $\boldsymbol{a}$ is defined to be

$$
\prod_{n \in I} a_{n}:=\exp \left(-\operatorname{Res}_{s=0} \frac{\zeta_{\boldsymbol{a}}(s)}{s^{2}}\right) .
$$

We use this dotted product symbol if $\zeta_{\boldsymbol{a}}(s)$ has a pole at $s=0$ in order to distinguish this notion from the standard holomorphic regularization. When $\zeta_{\boldsymbol{a}}(s)$ is holomorphic at $s=0$, it is elementary to check that $\zeta_{\boldsymbol{a}}^{\prime}(0)=\operatorname{Res}_{s=0} \zeta_{\boldsymbol{a}}(s) / s^{2}$, or

$$
\prod_{n \in I} a_{n}=\prod_{n \in I} a_{n} .
$$

The purpose of the present note is to provide explicit calculations of various examples which need the generalized notion above of the zeta regularized product and to observe certain transformation properties which are possessed by the functions defined by the zeta regularized products.

Remark 1.1. In [5], they deal with a wider class of regularization methods called " $\delta$-regularized products" which includes our generalized zeta regularization as a special case. In particular, they give a similar description of the relation between the (generalized) regularized product and the canonical form, which is in deed a generalization of the result in [18].

It is clear that (1.3) and (1.4) are still true in the case of this new regularization, but the formula (1.5) needs a slight modification as follows.

Lemma 1.1. The reguralized product of $\tau \boldsymbol{a}=\left\{\tau a_{n}\right\}_{n \in I}$ is given by

$$
\prod_{n \in I} \tau a_{n}=\exp \left(\sum_{l=1}^{N+1} \frac{(-1)^{l-1}}{l !}(\log \tau)^{l} \operatorname{Res}_{s=0} \zeta_{\boldsymbol{a}}(s) s^{l-2}\right) \times \prod_{n \in I} a_{n},
$$

where $N$ is the order of the pole of $\zeta_{\boldsymbol{a}}(s)$ at $s=0$.

Proof. In fact, if the Laurent expansion of $\zeta_{\boldsymbol{a}}(s)$ around $s=0$ is given by $\zeta_{\boldsymbol{a}}(s)=\sum_{k=-N}^{\infty} c_{k} s^{k}$, then we have

$$
\zeta_{\tau \boldsymbol{a}}(s)=\sum_{n}\left(\tau a_{n}\right)^{-s}=\tau^{-s} \zeta_{\boldsymbol{a}}(s)=\sum_{l=0}^{\infty} \frac{(-1)^{l}}{l !}(\log \tau)^{l} s^{l} \sum_{k=-N}^{\infty} c_{k} s^{k},
$$


whence

$$
\operatorname{Res}_{s=0} \frac{\zeta_{\tau \boldsymbol{a}}(s)}{s^{2}}=\sum_{l=0}^{N+1} \frac{(-1)^{l}}{l !}(\log \tau)^{l} c_{1-l}=\sum_{l=1}^{N+1} \frac{(-1)^{l}}{l !}(\log \tau)^{l} \operatorname{Res}_{s=0} \zeta_{\boldsymbol{a}}(s) s^{l-2}+\operatorname{Res}_{s=0} \frac{\zeta_{\boldsymbol{a}}(s)}{s^{2}} .
$$

In Section 2 we observe several arithmetic examples concerning the (generalized) regularized products of positive integers. In Section 3 we give several examples of functions defined by the regularized products.

\section{Some arithmetic examples}

As we can see typically in Riemann's calculation (1.2), the zeta regularized product of positive integers is one of the most interesting situation from the arithmetic point of view. Since we deal with the sequence of positive integers, it is convenient to use the convention

$$
\prod_{n \in I} a_{n}=\prod_{n=1}^{\infty}(n ; m(n))
$$

where $m(n)$ denotes the multiplicity of $n$ appearing in the sequence $\boldsymbol{a}=\left\{a_{n}\right\}_{n \in I}$, that is, the attached zeta function of $\boldsymbol{a}$ is

$$
\zeta_{\boldsymbol{a}}(s)=\sum_{n \in I} a_{n}^{-s}=\sum_{n=1}^{\infty} \frac{m(n)}{n^{s}} .
$$

By this notation we have for instance

$$
\begin{aligned}
& \prod_{n=1}^{\infty}(n ; 1)=\exp \left(-\zeta^{\prime}(0)\right)=\sqrt{2 \pi}, \\
& \prod_{n=1}^{\infty}(n ; n)=\exp \left(-\zeta^{\prime}(-1)\right), \\
& \prod_{n=1}^{\infty}\left(n ; n^{2}\right)=\exp \left(-\zeta^{\prime}(-2)\right), \quad \text { etc. }
\end{aligned}
$$

Now let us consider the case of square-free integers. Then we have remarkable identities

$$
\prod_{n: \text { square-free }} n=2 \pi=\prod_{n: \text { square }} n .
$$

In fact, the first equality is valid since we see that

$$
\sum_{n: \text { square-free }} n^{-s}=\prod_{p: \text { prime }}\left(1+p^{-s}\right)=\prod_{p: \text { prime }} \frac{1-p^{-2 s}}{1-p^{-s}}=\frac{\zeta(s)}{\zeta(2 s)}
$$


and

$$
\left.\frac{d}{d s}\left(\sum_{n: \text { square-free }} n^{-s}\right)\right|_{s=0}=\left.\frac{d}{d s}\left(\frac{\zeta(s)}{\zeta(2 s)}\right)\right|_{s=0}=\frac{\zeta^{\prime}(0) \zeta(0)-2 \zeta(0) \zeta^{\prime}(0)}{\zeta(0)^{2}}=-\log (2 \pi) .
$$

The second equality follows immediately from (1.2) and (1.4).

Next we look at the case of the regularized product of the square-free positive integers $n$ with having $n$ as its multiplicity, that is (if any)

$$
\prod_{n: \text { square-free }}(n ; n)
$$

Then we must study the behavior of the attached zeta function

$$
\varphi(s)=\sum_{n: \text { square-free }} \frac{n}{n^{s}}=\frac{\zeta(s-1)}{\zeta(2 s-2)}
$$

at $s=0$. Note here that $\varphi(s)$ is not holomorphic at the origin, and actually it has a simple pole there. Hence the regularized product does not exist in the original sense.

Thus we face with the situation requiring the extended notion of the zeta regularized product. In fact, by using the generalized zeta regularization, we have the following formulas.

THEOREM 2.1. We use a convention similar to (2.1) for generalized zeta regularized products.

(i) The regularized product over the square-free positive integers $n$ with multiplicity $n$ is given by

$$
\begin{aligned}
& \prod_{n: s q u a r e-f r e e}(n ; n) \\
= & { }^{\prime} 1 \times 2 \times 2 \times 3 \times 3 \times 3 \times 5 \times 5 \times 5 \times 5 \times 5 \times 6 \times 6 \times 6 \times 6 \times 6 \times 6 \times \ldots, \\
= & \exp \left(-\frac{1}{4} \frac{\zeta^{\prime \prime}(-1)}{\zeta^{\prime}(-2)}+\frac{1}{2} \frac{\zeta^{\prime}(-1) \zeta^{\prime \prime}(-2)}{\zeta^{\prime}(-2)^{2}}-\frac{1}{36} \frac{\zeta^{\prime \prime \prime}(-2)}{\zeta^{\prime}(-2)^{2}}+\frac{1}{24} \frac{\zeta^{\prime \prime}(-2)^{2}}{\zeta^{\prime}(-2)^{3}}\right) .
\end{aligned}
$$

(ii) We denote by $\mu(n)$ the usual Möbius function. Then we have

$$
\begin{aligned}
\prod_{\mu(n)=+1}\left(n ; n^{2}\right)=\exp ( & \frac{1}{12} \frac{\zeta^{\prime \prime \prime}(-2)}{\zeta^{\prime}(-2)^{2}}-\frac{1}{8} \frac{\zeta^{\prime \prime}(-2)^{2}}{\zeta^{\prime}(-2)^{3}} \\
& \left.+\frac{1}{4} \frac{\zeta^{\prime}(-2) \zeta^{\prime \prime}(-4)}{\zeta^{\prime}(-4)^{2}}-\frac{1}{8} \frac{\zeta^{\prime \prime}(-2)}{\zeta^{\prime}(-4)}\right),
\end{aligned}
$$




$$
\begin{aligned}
\prod_{\mu(n)=-1}\left(n ; n^{2}\right)=\exp ( & -\frac{1}{12} \frac{\zeta^{\prime \prime \prime}(-2)}{\zeta^{\prime}(-2)^{2}}+\frac{1}{8} \frac{\zeta^{\prime \prime}(-2)^{2}}{\zeta^{\prime}(-2)^{3}} \\
& \left.+\frac{1}{4} \frac{\zeta^{\prime}(-2) \zeta^{\prime \prime}(-4)}{\zeta^{\prime}(-4)^{2}}-\frac{1}{8} \frac{\zeta^{\prime \prime}(-2)}{\zeta^{\prime}(-4)}\right) .
\end{aligned}
$$

Proof. (i) By (2.7) the attached zeta function is $\zeta(s-1) / \zeta(2 s-2)$. Remarking that $\zeta(-1)=-1 / 12$ and $\zeta(-2)=0$ with $\zeta^{\prime}(-2) \neq 0$, we have the Laurent expansion around $s=0$ as

$$
\begin{aligned}
& \zeta(s-1)=-\frac{1}{12}+s \zeta^{\prime}(-1)+\frac{s^{2}}{2} \zeta^{\prime \prime}(-1)+O\left(s^{3}\right), \\
& \frac{1}{\zeta(2 s-2)}=\frac{1}{s} \frac{1}{2 \zeta^{\prime}(-2)}-\frac{1}{2} \frac{\zeta^{\prime \prime}(-2)}{\zeta^{\prime}(-2)^{2}}+2 s\left(-\frac{1}{6} \frac{\zeta^{\prime \prime \prime}(-2)}{\zeta^{\prime}(-2)^{2}}+\frac{1}{4} \frac{\zeta^{\prime \prime}(-2)}{\zeta^{\prime}(-2)^{3}}\right)+O\left(s^{2}\right),
\end{aligned}
$$

and hence

$$
\begin{aligned}
\frac{\zeta(s-1)}{\zeta(2 s-2)}= & \frac{1}{s}\left(-\frac{1}{24 \zeta^{\prime}(-2)}\right)+\left(\frac{1}{2} \frac{\zeta^{\prime}(-1)}{\zeta^{\prime}(-2)}+\frac{1}{24} \frac{\zeta^{\prime \prime}(-2)}{\zeta^{\prime}(-2)^{2}}\right) \\
+ & s\left(-\frac{1}{4} \frac{\zeta^{\prime \prime}(-1)}{\zeta^{\prime}(-2)}+\frac{1}{2} \frac{\zeta^{\prime}(-1) \zeta^{\prime \prime}(-2)}{\zeta^{\prime}(-2)^{2}}\right. \\
& \left.-\frac{1}{36} \frac{\zeta^{\prime \prime \prime}(-2)}{\zeta^{\prime}(-2)^{2}}+\frac{1}{24} \frac{\zeta^{\prime \prime}(-2)^{2}}{\zeta^{\prime}(-2)^{3}}\right)+O\left(s^{2}\right) .
\end{aligned}
$$

Thus we have (2.8).

(ii) Since we have

$$
\begin{aligned}
\sum_{n: \text { square-free }} n^{-s} & =\frac{\zeta(s)}{\zeta(2 s)}, \\
\sum_{n \text { :square-free }} \mu(n) n^{-s} & =\frac{1}{\zeta(s)},
\end{aligned}
$$

we obtain

$$
\begin{aligned}
\sum_{\mu(n)=+1} n^{-s} & =\frac{1}{2}\left(\frac{1}{\zeta(s)}+\frac{\zeta(s)}{\zeta(2 s)}\right), \\
\sum_{\mu(n)=-1} n^{-s} & =\frac{1}{2}\left(-\frac{1}{\zeta(s)}+\frac{\zeta(s)}{\zeta(2 s)}\right) .
\end{aligned}
$$

Thus the attached zeta functions are given by $(1 / 2)(1 / \zeta(s-2)+\zeta(s-2) /$ 
$\zeta(2 s-4))$ and $(1 / 2)(-1 / \zeta(s-2)+\zeta(s-2) / \zeta(2 s-4))$ respectively. Now the statements (2.9) and (2.10) follow from the Laurent expansions

$$
\begin{aligned}
& \frac{1}{\zeta(s-2)}=\frac{1}{s} \frac{1}{\zeta^{\prime}(-2)}-\frac{1}{2} \frac{\zeta^{\prime \prime}(-2)}{\zeta^{\prime}(-2)^{2}}+s\left(-\frac{1}{6} \frac{\zeta^{\prime \prime \prime}(-2)}{\zeta^{\prime}(-2)^{2}}+\frac{1}{4} \frac{\zeta(-2)^{2}}{\zeta^{\prime}(-2)^{3}}\right)+O\left(s^{2}\right), \\
& \frac{\zeta(s-2)}{\zeta(2 s-4)}=\frac{\zeta^{\prime}(-2)}{2 \zeta^{\prime}(-4)}+s\left(-\frac{1}{2} \frac{\zeta^{\prime}(-2) \zeta^{\prime \prime}(-4)}{\zeta^{\prime}(-4)^{2}}+\frac{1}{4} \frac{\zeta^{\prime \prime}(-2)}{\zeta^{\prime}(-4)}\right)+O\left(s^{2}\right) .
\end{aligned}
$$

This ends the proof of the theorem.

COROLlary 2.2. We have

$$
\prod_{n=1}^{\infty}\left(n ; n^{2}(1+\mu(n))=\exp \left(-\zeta^{\prime}(-2)+\frac{1}{6} \frac{\zeta^{\prime \prime \prime}(-2)}{\zeta^{\prime}(-2)^{2}}-\frac{1}{4} \frac{\zeta^{\prime \prime}(-2)^{2}}{\zeta^{\prime}(-2)^{3}}\right) .\right.
$$

Proof. Since the left hand side is given by the product of

$$
\prod_{\mu(n)=0}\left(n ; n^{2}\right)=\frac{\prod_{n=1}^{\infty}\left(n ; n^{2}\right)}{\prod_{n: \text { square-free }}\left(n ; n^{2}\right)}=\frac{\prod_{n=1}^{\infty}\left(n ; n^{2}\right)}{\prod_{\mu(n)=+1}\left(n ; n^{2}\right) \prod_{\mu(n)=-1}\left(n ; n^{2}\right)}
$$

and

$$
\bigoplus_{\mu(n)=+1}\left(n ; 2 n^{2}\right)=\left(\bigoplus_{\mu(n)=+1}\left(n ; n^{2}\right)\right)^{2},
$$

the result follows from (2.5) and (2.9), (2.10).

Remark 2.1. A divergent product " $1 \times 2 \times 2 \times 3 \times 3 \times 3 \times 4 \times 4 \times 4 \times 4 \times$ ..." allows us naively two kinds of interpretation as

$$
\prod_{n=1}^{\infty}(n ; n), \quad \prod_{n=1}^{\infty} n^{n} .
$$

Though the former regularized product exists in the sense of holomorphic zeta regularization, the latter does not exists even in the sense of generalized zeta regularization.

\section{Functions defined via zeta regularization}

We give here several examples of functions defined by generalized zeta regularized products. First we see the simplest example we does need the generalized zeta regularization. 
EXAMPLE 3.1. For any $q>1$, we have

$$
\prod_{n=0}^{\infty} q^{n+x}=q^{-B_{2}(x) / 2}
$$

where $B_{2}(x)$ is the Bernoulli polynomial of degree 2. This follows from the Laurent expansion of the zeta function for $\boldsymbol{a}=\left\{q^{n+x}\right\}_{n \geq 0}$;

$$
\zeta_{\boldsymbol{a}}(s, x)=\sum_{n=0}^{\infty} q^{-s(n+x)}=\frac{q^{-s x}}{1-q^{-s}}=\frac{1}{s \log q}+B_{1}(x)+\frac{s}{2} B_{2}(x) \log q+O\left(s^{2}\right) .
$$

This is interpreted as an exponential version of the special value formula

$$
\zeta(-1, x)=-\frac{B_{2}(x)}{2}
$$

of the Hurwitz zeta function $\zeta(s, x)=\sum_{n=0}^{\infty}(n+x)^{-s}$.

The following example is an analogues (or generalized) situation of the example above.

EXAmPle 3.2. Let $a>1$. For $0<x<1$, we have

$$
\prod_{n \geq 0}\left(a^{n}-x\right)=a^{-1 / 12} \prod_{n \geq 0}\left(1-\frac{x}{a^{n}}\right) .
$$

In fact, the attached zeta function is calculated as follows:

$$
\begin{aligned}
\zeta_{\boldsymbol{a}}(s, x) & =\sum_{n=0}^{\infty}\left(a^{n}-x\right)^{-s}=\sum_{n=0}^{\infty} a^{-n s} \sum_{l=0}^{\infty}\left(\begin{array}{c}
s+l-1 \\
l
\end{array}\right)\left(\frac{x}{a^{n}}\right)^{l} \\
& =\frac{1}{s \log a}+\frac{1}{2}+\frac{s \log a}{12}-s \log \prod_{n=0}^{\infty}\left(1-\frac{x}{a^{n}}\right)+O\left(s^{2}\right) .
\end{aligned}
$$

\subsection{Analogues of Lerch's formula}

The next example is a $q$-analogue of Lerch's formula (1.6), which allows us to calculate in the sense of generalized reguralized product.

EXAMPLE 3.3 ( $q$-Lerch's formula [13]). We employ the following convention for the $q$-analogue of numbers:

$$
[a]_{q}:=\frac{q^{a / 2}-q^{-a / 2}}{q^{1 / 2}-q^{-1 / 2}} \quad(a \in \boldsymbol{C}) .
$$

A $q$-analogue version of Lerch's formula (1.6) is

$$
\prod_{n=0}^{\infty}[n+x]_{q}=\frac{[\infty]_{q} !}{\Gamma_{q}(x)}
$$


Here we denote by $\Gamma_{q}(x)$ the (modified) Jackson $q$-gamma function

$$
\Gamma_{q}(x):=\frac{\prod_{n=1}^{\infty}\left(1-q^{-n}\right)}{\prod_{n=0}^{\infty}\left(1-q^{-(n+x)}\right)}\left(q^{1 / 2}-q^{-1 / 2}\right)^{1-x} q^{x(x-1) / 4} .
$$

The constant $[\infty]_{q}$ ! is given explicitly by

$$
[\infty]_{q} !:=\prod_{n=1}^{\infty}[n]_{q}=q^{-1 / 24}\left(q^{1 / 2}-q^{-1 / 2}\right)^{-\log \left(1-q^{-1}\right) / \log q} \prod_{n=1}^{\infty}\left(1-q^{-n}\right) .
$$

Remark 3.1. The present convention of $q$-numbers $[a]_{q}$ is different from the one used in [13].

EXAMPLE 3.4. We have

$$
\bigoplus_{m, n \geq 1}(m n-x)=\frac{1}{\sqrt{2 \pi}} e^{-\left(\gamma^{2}+2 \gamma_{1}\right) x} \prod_{m, n \geq 1}\left(1-\frac{x}{m n}\right) e^{x / m n},
$$

where $\gamma$ is the Euler constant and $\gamma_{1}$ is the higher Euler constant (see, e.g. [4]), that is, the coefficient of the linear term in the Laurent expansion of $\zeta(s)$ around $s=1$ :

$$
\zeta(s)=\frac{1}{s-1}+\gamma+\gamma_{1}(s-1)+O\left(s^{2}\right) .
$$

In fact, the attached zeta function is calculated as follows:

$$
\begin{aligned}
\zeta_{\boldsymbol{a}}(s, x)= & \sum_{m, n \geq 1}(m n-x)^{-s}=\zeta(s)^{2}+s \zeta(s+1)^{2} x \\
& +s \sum_{l=2}^{\infty} \frac{1}{l} \zeta(s+l)^{2} x^{l}+O\left(s^{2}\right)=\frac{x}{s}+\left(\zeta(0)^{2}-2 \gamma x\right) \\
& +\left(2 \zeta(0) \zeta^{\prime}(0)+\left(\gamma^{2}+2 \gamma_{1}\right) x+\sum_{l=2}^{\infty} \frac{1}{l} \zeta(l)^{2} x^{l}\right) s+O\left(s^{2}\right) .
\end{aligned}
$$

Therefore the formula above follows from the special values of the Riemann zeta function $\zeta(0)=-1 / 2, \zeta^{\prime}(0)=-(1 / 2) \log 2 \pi$.

EXAMPLE 3.5. Let $a$ be a positive number and $m$ a positive integer. Then we have

$$
\begin{aligned}
& \bigoplus_{n \geq 1}\left((n+x)^{a+1 / m}-(n+x)^{a}\right) \\
& \quad=\exp \left(\left(a+\frac{1}{m}\right)^{-1} \sum_{0<i<j<m} \frac{1}{i j}+\sum_{\substack{l>0 \\
l \neq m}} \frac{1}{l} \zeta\left(\frac{l}{m}, x\right)-\frac{1}{m} \frac{\Gamma^{\prime}(x)}{\Gamma(x)}\right)\left(\frac{\sqrt{2 \pi}}{\Gamma(x)}\right)^{a+1 / m} .
\end{aligned}
$$


In fact, the attached zeta function is calculated as follows:

$$
\zeta_{\boldsymbol{a}}(s, x)=\sum_{n=0}^{\infty}\left((n+x)^{a+1 / m}-(n+x)^{a}\right)^{-s}=\sum_{l=0}^{\infty}\left(\begin{array}{c}
s+l-1 \\
l
\end{array}\right) \zeta\left(s a+\frac{s+l}{a}\right) .
$$

Remark that (3.12) is also true for $a=0$, and it gives the Lerch formula (1.6) when $m=1$.

\subsection{Multiplicative anomaly}

In general we can not expect the multiplication law of the regularized product

$$
\prod_{n \in I} a_{n} \prod_{n \in I} b_{n} \stackrel{?}{=} \prod_{n \in I} a_{n} b_{n},
$$

even if all these three products exist. In fact,

$$
\operatorname{Res}_{s=0} \frac{\zeta_{\boldsymbol{a}}(s)}{s^{2}}+\operatorname{Res}_{s=0} \frac{\zeta_{\boldsymbol{b}}(s)}{s^{2}}-\operatorname{Res}_{s=0} \frac{\zeta_{\boldsymbol{a}}(s)}{s^{2}}
$$

is not identically zero in general, hence yields the multiplicative anomaly even in the holomorphic regularized products case. Here $\zeta_{\boldsymbol{a b}}(s)$ is the zeta function associated with the pointwise product sequence $\boldsymbol{a} \boldsymbol{b}=\left\{a_{n} b_{n}\right\}_{n \in I}$ of $\boldsymbol{a}=\left\{a_{n}\right\}_{n \in I}$ and $\boldsymbol{b}=\left\{b_{n}\right\}_{n \in I}$. See e.g., [8], [17].

From this point of view, here we give two examples of regularized products. The first example is a regularized product expression of the Beta function, which has no multiplicative anomaly.

EXAMPLE 3.6 (Beta function). The following equalities hold.

$$
\prod_{n=0}^{\infty} \frac{(x+n)(y+n)}{x+y+n}=\sqrt{2 \pi} \frac{\Gamma(x+y)}{\Gamma(x) \Gamma(y)}=\frac{\prod_{n=0}^{\infty}(x+n) \prod_{n=0}^{\infty}(y+n)}{\prod_{n=0}^{\infty}(x+y+n)} .
$$

Notice that $\arg ((x+n)(y+n) /(x+y+n))=\arg (x+n)+\arg (y+n)-$ $\arg (x+y+n)$ for every $n \geq 0$. Here, for simplicity, we assume $0<\operatorname{Re} x<1$, $0<\operatorname{Re} y<1,0<\operatorname{Re}(x+y)<1$, which assure us to apply the binomial theorem for all $n=1,2, \ldots$ in the following calculation. The corresponding zeta function is calculated as follows.

$$
\begin{aligned}
\beta(s, x, y) & :=\sum_{n=0}^{\infty}\left\{\frac{(x+n)(y+n)}{x+y+n}\right\}^{-s} \\
& =\left(\frac{x y}{x+y}\right)^{-s}+\sum_{n=1}^{\infty} n^{-s}\left(1+\frac{x}{n}\right)^{-s}\left(1+\frac{y}{n}\right)^{-s}\left(1+\frac{x+y}{n}\right)^{s}
\end{aligned}
$$


SOME EXAMPLES OF GENERALIZED ZETA REGULARIZED PRODUCTS

$$
\begin{aligned}
= & \left(\frac{x y}{x+y}\right)^{-s}+\sum_{k, \ell, m=0}^{\infty}\left(\begin{array}{c}
-s \\
k
\end{array}\right)\left(\begin{array}{c}
-s \\
\ell
\end{array}\right)\left(\begin{array}{c}
s \\
m
\end{array}\right) x^{k} y^{\ell}(x+y)^{m} \zeta(k+\ell+m+s) \\
= & \frac{1}{2}-\left\{\log \left(\frac{x y}{x+y}\right)+\frac{1}{2}(\log 2 \pi)\right\} s+\sum_{k=1}^{\infty}\left(\begin{array}{c}
-s \\
k
\end{array}\right) x^{k} \zeta(k+s) \\
& +\sum_{\ell=1}^{\infty}\left(\begin{array}{c}
-s \\
\ell
\end{array}\right) y^{\ell} \zeta(\ell+s)+\sum_{m=1}^{\infty}\left(\begin{array}{c}
s \\
m
\end{array}\right)(x+y)^{m} \zeta(m+s)+O\left(s^{2}\right) \\
= & \frac{1}{2}-s \log \frac{1}{\sqrt{2 \pi}} \frac{\Gamma(x) \Gamma(y)}{\Gamma(x+y)}+O\left(s^{2}\right) .
\end{aligned}
$$

The latter equality follows from the Lerch formula (1.6).

The next example is a $q$-analogue of the example above, which possesses a slight anomaly.

EXAMPLE 3.7 ( $q$-Beta function). The following equalities hold.

$$
\prod_{n=0}^{\infty} \frac{[x+n]_{q}[y+n]_{q}}{[x+y+n]_{q}}=\frac{[\infty]_{q} !}{q^{(1 / 2) x y}} \frac{\Gamma_{q}(x+y)}{\Gamma_{q}(x) \Gamma_{q}(y)}=q^{-(1 / 2) x y} \frac{\prod_{n=0}^{\infty}[x+n]_{q} \prod_{n=0}^{\infty}[y+n]_{q}}{\prod_{n=0}^{\infty}[x+y+n]_{q}} .
$$

In fact, the corresponding zeta function is given by

$$
\begin{aligned}
\beta_{q}(s, x, y): & =\sum_{n=0}^{\infty}\left\{\frac{[x+n]_{q}[y+n]_{q}}{[x+y+n]_{q}}\right\}^{-s} \\
= & \left(q^{1 / 2}-q^{-1 / 2}\right)^{s} \sum_{n=0}^{\infty} q^{-(1 / 2) n s}\left(1-q^{-x-n}\right)^{-s}\left(1-q^{-y-n}\right)^{-s}\left(1-q^{-x-y-n}\right)^{s} \\
= & \left(q^{1 / 2}-q^{-1 / 2}\right)^{s}\left\{\frac{2}{\log q} \frac{1}{s}+\frac{1}{2}+\left(\frac{1}{24} \log q+\sum_{k=1}^{\infty} \frac{q^{k(1-x)}}{k\left(q^{k}-1\right)}\right.\right. \\
& \left.\left.\quad+\sum_{\ell=1}^{\infty} \frac{q^{\ell(1-y)}}{\ell\left(q^{\ell}-1\right)}-\sum_{m=1}^{\infty} \frac{q^{m(1-x-y)}}{m\left(q^{m}-1\right)}\right) s+O\left(s^{2}\right)\right\} \\
= & \frac{2}{\log q} \frac{1}{s}+\frac{1}{2}+\log \left(q^{1 / 2}-q^{-1 / 2}\right)+s \log \left(\frac{q^{(1 / 2) x y}}{[\infty]_{q} !} \frac{\Gamma_{q}(x) \Gamma_{q}(y)}{\Gamma_{q}(x+y)}\right)+O\left(s^{2}\right) .
\end{aligned}
$$

In the calculation procedure above we have assumed that $0<\operatorname{Re} x<1,0<$ $\operatorname{Re} y<1,0<\operatorname{Re}(x+y)<1$ for the same reason as in Example 3.6. We note 
that the cross term $q^{-(1 / 2) x y}$ comes from the quadratic factor $q^{x(x-1) / 4}$ in the definition of $\Gamma_{q}(x)$. Moreover, the latter equality follows immediately from Lerch's formula (3.7) and hence the anomaly is given by $q^{-(1 / 2) x y}$.

Remark 3.2. The above type formulas hold also for the so-called Selberg (resp. q-Selberg) type integrals if we use their expressions by means of the products and quotients of gamma (resp. q-gamma) functions.

Remark 3.3. In contrast with the case of the Kronecker limit formula (see [9]), the product of the abosolute values of the factor $|(x+n)(y+n) /(x+y+n)|$ (resp. $\left.\left|[x+n]_{q}[y+n]_{q} /[x+y+n]_{q}\right|\right)$ is equal to the absolute value of the product. For instance, we have

$$
\prod_{n=0}^{\infty}\left|\frac{[x+n]_{q}[y+n]_{q}}{[x+y+n]_{q}}\right|=\left|\prod_{n=0}^{\infty} \frac{[x+n]_{q}[y+n]_{q}}{[x+y+n]_{q}}\right| .
$$

This is just because the products in these cases are taken over the semi-lattice of non-negative integers which are not the all integers in the cases of the Kronecker limit formula and its $q$-analogue (see also [7]).

If we look at the difference of the zeta functions $\zeta_{\boldsymbol{a} \boldsymbol{b}}(s)$ and $\zeta_{\boldsymbol{a}}(s) \zeta_{\boldsymbol{b}}(s)$, we have for instance the following.

EXAMPLE 3.8. We have

$$
\bigoplus_{m \neq n \geq 1}(m-x)(n+x)=\frac{1}{2 \pi}\{\Gamma(x) \Gamma(-x)\}^{1 / 2}\left\{\frac{\Gamma(x)}{\Gamma(-x)}\right\}^{x} .
$$

It is not hard to see that this product defines a meromorphic (single valued) function in the whole $\boldsymbol{C}$.

\subsection{Transformation properties}

In a last position of the present note, we show the multiplication formula of $\Gamma(x)$ by using (1.3) (not (3.14)) and (1.5).

EXAMPLE 3.9 (Multiplication formula of Gauss-Legendre). The gamma function $\Gamma(x)$ satisfies the multiplication formula

$$
\Gamma(N x)=\frac{N^{N x}}{(2 \pi)^{(N-1) / 2} \sqrt{N}} \Gamma(x) \Gamma\left(x+\frac{1}{N}\right) \Gamma\left(x+\frac{2}{N}\right) \cdots \Gamma\left(x+\frac{N-1}{N}\right) .
$$

In fact, it is immediate from Lerch's formula that 
SOME EXAMPLES OF GENERALIZED ZETA REGULARIZED PRODUCTS

$$
\begin{aligned}
\frac{\sqrt{2 \pi}}{\Gamma(N x)} & =\prod_{m=0}^{\infty}(m+N x)=\prod_{k=0}^{N-1} \prod_{m=0}^{\infty}((m N+k)+N x) \\
& =\prod_{k=0}^{N-1} \prod_{m=0}^{\infty} N\left(m+\left(x+\frac{k}{N}\right)\right) \\
& =\prod_{k=0}^{N-1} N^{\zeta(0, x+k / N)} \prod_{m=0}^{\infty}\left(m+\left(x+\frac{k}{N}\right)\right)=\prod_{k=0}^{N-1} N^{1 / 2-(x+k / N)} \frac{\sqrt{2 \pi}}{\Gamma(x+k / N)} \\
& =\sqrt{2 \pi}^{N} N^{1 / 2-N x} \prod_{k=0}^{N-1} \frac{1}{\Gamma(x+k / N)},
\end{aligned}
$$

which is the desired formula.

The next example seems also significant in view of the transformation property (1.13).

EXAMPLE 3.10. Let $q>1$ be a fixed parameter. Then we have

$$
\begin{aligned}
\prod_{n \in \boldsymbol{Z}}\left(q^{n} x+q^{-n}\right)= & q^{-1 / 6}\left(\sqrt{x}+\sqrt{x}^{-1}\right) \exp \left(-\frac{1}{2} \frac{(\log x)^{2}}{\log q}\right) \\
& \times \prod_{n=1}^{\infty}\left(1+x q^{-2 n}\right)\left(1+x^{-1} q^{-2 n}\right) .
\end{aligned}
$$

In fact, the attached zeta function is calculated as follows (here we assume that $q^{-2}<|x|<q^{2}$ for a technical reason):

$$
\zeta_{\boldsymbol{a}}(s, x)=(x+1)^{-s}+\varphi(s, x)+x^{-s} \varphi\left(s, x^{-1}\right),
$$

where

$$
\begin{aligned}
\varphi(s, x) & =\sum_{n=1}^{\infty} q^{-n s}\left(1+x q^{-2 n}\right)^{-s} \\
& =\frac{1}{s \log q}-\frac{1}{2}+\frac{s \log q}{12}+\sum_{l=1}^{\infty} \frac{(-1)^{l}}{l} \frac{x^{l}}{q^{2 l}-1} s+O\left(s^{2}\right) .
\end{aligned}
$$

The statement follows from the following identity

$$
\sum_{l=1}^{\infty} \frac{(-1)^{l}}{l} \frac{x^{l}}{q^{l}-1}=-\log \prod_{n=1}^{\infty}\left(1+x q^{-n}\right) .
$$

It is worth noting that since

$$
\prod_{n \in \boldsymbol{Z}}\left(q^{n} x^{-1}+q^{-n}\right)=\prod_{n \in \boldsymbol{Z}} \frac{\left(q^{n} x+q^{-n}\right)}{x},
$$


once we know the existence of the regularized product, we can find that this function is invariant under the map $x \leftrightarrow 1 / x$ by (1.13) in Lemma 1.1. Moreover, the expression (3.19) shows that the regularized product possesses the information of location of zeros.

Remark 3.4. In [7], the generalized regularized product

$$
\prod_{n=1}^{\infty} \frac{\sinh (n+x)}{\sinh (x)}
$$

is essentially calculated and one knows then it gives the double sine function $S_{2}(x)$ (which is the elliptic theta function up to the elementary factor). From this point of view, it is a natural and an important problem to calculate the regularized product

$$
" \prod_{n=1}^{\infty} \frac{\Gamma(n+x)}{\Gamma(x)} "
$$

if it exists, and this is expected to give essentially the double gamma function $\Gamma_{2}(x)$ (see [1], also [10], [11]). Notice that in view of the reflection formula $S_{2}(x)=\Gamma_{2}(x)^{-1} \Gamma_{2}(2-x)$, the product (3.25) is actually regarded as the "half" of (3.24). We remark also that these kinds of products (3.24), (3.25) are quite useful for the study of zeta extensions [14].

It is also interesting to study

$$
\prod_{n=0}^{\infty} q^{(n+x)^{k} "}
$$

for $k \geq 2$. This is a natural generalization of (3.1), and hence we expect to have a proper situation that (3.26) may give $q^{-B_{1+k}(x) /(1+k)}$.

Unfortunately, the generalized regularization method does not work in these cases since the attached zeta functions are not meromorphic at the origin $s=0$. Thus we hope to formulate the further generalization of zeta regularization which makes us possible to deal with wider class of divergent products including (3.25), (3.26) and the $q$-analogue of the results developed in [12].

\section{REFERENCES}

[1] E. W. Barnes, On the theory of the multiple gamma function, Trans. Cambridge Philos. Soc. 19 (1904), 374-425.

[2] C. Deninger, Local $L$-factors of motives and regularized determinants, Invent. math. 107 (1992), 135-150.

[ 3 ] M. Lerch, Dalši studie v oboru Malmsténovských řad, Rozpravy České Akad. 3 (1894), No. 28, 1-61.

[ 4 ] Y. Hashimoto, Y. Iijima, N. Kurokawa and M. Wakayama, Euler's constants for the Selberg and the Dedekind zeta functions, to appear in Bull. Belg. Math. Soc. Simon Stevin. 
[ 5 ] G. ILlies, Regularized products and determinants, Commun. Math. Phys. 220 (2001), 69-94.

[6] M. KaneKo, N. Kurokawa and M. Wakayama, A variation of Euler's approach to values of the Riemann zeta function, Kyushu J. Math. 57 (2003), 175-192.

[7] K. Kimoto, N. Kurokawa, C. Sonoki and M. Wakayama, Zeta regularizations and $q$ analogue of ring sine functions, Kyushu J. Math. 57 (2003), 197-215.

[8] M. Kontsevich AND S. VishiK, Geometry of determinants of elliptic operators, Functional analysis on the eve of the 21st century, Vol. 1 (New Brunswick, NJ, 1993), Progr. Math. 131, Birkhäuser Boston, Boston, MA, 1995, 173-197.

[9] N. Kurokawa, E. M. Müller-Stüler, H. Ochiai and M. Wakayama, Kronecker's Jugendtraum and ring sine functions, J. Ramanujan Math. Soc. 17 (2002), 211-220.

[10] N. Kurokawa and S. Koyama, Multiple sine functions, Forum. Math. 15 (2003), 839-876.

[11] N. Kurokawa, H. Ochiai and M. Wakayama, Multiple Trigonometry and zeta functions, J. Ramanujan Math. Soc. 17 (2002), 101-113.

[12] N. Kurokawa AND M. WaKayama, A generalization of Lerch's formula, to appear in Czech. Math. J.

[13] N. Kurokawa and M. Wakayama, Generalized zeta regularizations, quantum class number formulas, and Appell's $O$-functions, to appear in The Ramanujan $\mathbf{J}$.

[14] N. Kurokawa and M. Wakayama, Zeta extensions, Proc. Japan. Acad. Ser. A 78 (2002), $126-130$.

[15] N. Kurokawa and M. Wakayama, On $q$-analogue of the Euler constant and Lerch's limit formula, Proc. Amer. Math. Soc. 132 (2004), 935-941.

[16] N. Kurokawa and M. Wakayama, Certain families of elliptic functions defined by $q$-series, to appear in The Ramanujan $\mathrm{J}$.

[17] J. R. Quine AND J. ChoI, Zeta regularized products and functional determinants on spheres, Rocky Mountain J. Math. 26 (1996), 719-729.

[18] A. Voros, Spectral functions, special functions and the Selberg zeta functions, Commun. Math. Phys. 110 (1987), 439-465.

\author{
KAZUFUMi Kimoto \\ Department of Mathematical Science, University of the Ryukyus \\ NishiHaRa, OKInAWa, 903-0231 JAPAN \\ kimoto@math.u-ryukyu.ac.jp \\ Nobushige KuRoKawa \\ Department of Mathematics, Tokyo Institute of Technology \\ MEguro, TOKYo, 152-8551 JAPAN \\ kurokawa@math.titech.ac.jp \\ ChIE Sonoki \\ NEC CORPORATION \\ 1131 Hinode, Abiko, ChiBA, 270-1198 JAPAN \\ c-sonoki@bx.jp.nec.com \\ Masato WaKayama \\ Faculty of Mathematics, KyUshu University \\ HaKozAKI, FuKUOKa, 812-8581 JAPAN \\ wakayama@math.kyushu-u.ac.jp
}

